\title{
Sample Transport Optimization: Mali Pilot Study
}

\author{
Hamadoun Kassambara, Marjorie Larson Nana, Famory Samassa, and Mamadou Diango Traoré
}

In Mali, qualified laboratories for testing of dangerous pathogens are centralized in Bamako. Creating a specimen transport system respecting timeline, specimen quality, biosafety, and biosecurity standards is a challenge. The current ad hoc system that relies on untrained public transport companies carries risks of spoilage, accidental release of pathogens, and delays, which compromise specimen quality. This pilot study aimed to evaluate the effectiveness (ie, timeline, quality of specimen, and cost) of using the trained postal service for sample transportation from district to central level, compared with the current system. The postal service intervention ran from mid-2016 to mid-2017 and covered 3 districts. Data were collected in the same districts during the same period of the preceding year for comparison. In all, 41 specimens were shipped using public transportation and 51 were shipped using the postal service. These included suspected meningitis, measles, yellow fever, and polio samples. Only $46 \%$ of samples sent by public transportation were received in Bamako within 72 hours of collection, compared to $71 \%$ of samples shipped via the postal service $(p<.05)$. Further, $93 \%$ of samples shipped by public transportation arrived in good condition at the receiving laboratory, compared to $98 \%$ by postal service. Although cost comparisons were difficult (flat fee vs per-specimen fare), the average cost per specimen was 8 times higher with the postal service. Shipment of specimens from districts to central level using the postal service was feasible and appeared to be faster than public transportation, thus allowing specimen quality to be preserved. Further analysis regarding the most efficient costing mechanism is needed.

Keywords: Public health preparedness/response, Specimen transport, Epidemic control

$\mathrm{T}$ he West Africa Ebola epidemic of 2014-15 revealed serious gaps in national capacities, from laboratories to surveillance to rapid response. In Liberia, poor infrastructure in isolated Ebola-affected communities led to serious delays in sending specimens, with no plan in place to surmount these challenges during an epidemic. ${ }^{1}$ It is difficult to quantify the extent to which suboptimal specimen transport systems contributed to degraded, damaged, lost, or delayed specimens arriving at testing facilities during the epidemic, due to multiple simultaneous health system weaknesses; nevertheless, it is a documented fact. ${ }^{2}$ Specimen transport systems are an under-recognized component of a country's laboratory capacity; inefficient specimen transport systems lead to long turnaround time and higher likelihood of loss or breakage in transit, which could be critical during a disease outbreak. ${ }^{3}$ This contributes to the widespread problem of delays in laboratory results in subSaharan Africa. ${ }^{4}$

Hamadoun Kassambara, MPH, is Chief of Party, GHSA, and Mamadou Diango Traoré, MS, is Data Manager, GHSA; both in the Department of Health, Catholic Relief Services, Bamako, Mali. Marjorie Larson Nana, MPH, is a Program Officer, Johns Hopkins Center for Communications Programs, Johns Hopkins University, Baltimore, MD. Famory Samassa, MPH, is Senior Technical Advisor for Health, Management Science for Health, Bamako, Mali. The findings and conclusions in this report are those of the authors and do not necessarily represent the official position of the US Centers for Disease Control and Prevention. 
The specimen transport system was integrated as a core component of laboratory capacity under the Global Health Security Agenda (GHSA), which assists countries in preventing, detecting, and responding to disease threats. As recommended by the Joint External Evaluation (JEE) tools of the International Health Regulations (IHR, 2005), minimum standards for specimen timeliness, tracking, training, and quality assurance should be consistently met across an increasing percentage of districts to increase a country's capacity score. ${ }^{5}$ One way to achieve this is to leverage existing logistical systems to improve standardization and efficiency. ${ }^{6}$ Public-private partnerships with private or parastatal logistical firms (such as many national postal companies) for specimen transport showed feasibility and positive effects on turnaround time in Ethiopia. ${ }^{7}$ However, literature on specimen transport systems in subSaharan Africa is sparse.

Mali's work on the specimen transport system began with initiation of GHSA activities in 2015. Until 2016, there was no official policy on transport of samples of epidemic-prone diseases. An ad hoc system was put into place in which public transport companies are reimbursed per specimen, based on distance between health districts and Bamako. All samples are brought to the National Health Directorate (DNS) in Bamako and then sent onward to the appropriate national reference laboratories or outside of Mali to World Health Organization (WHO) regional laboratories. The district-to-central level transport often involves poor conditions; samples may be transported along with other nonbiological items without sufficient isolation, on unpaved roads, leaving them vulnerable to breakage. Samples are handled by employees untrained to manage biohazard material. And buses very often face breakdowns and delays, increasing transit time.

Weaknesses inherent in this system were identified in an analysis of the specimen transport system carried out by the African Society for Laboratory Medicine (ASLM) in $2015 .{ }^{8}$ The analysis concluded that the specimen transport system had significant inefficiencies and was fragmented by disease (ie, donors fund shipment of TB samples or HIV samples, rather than taking a more integrated approach). To this challenge is added the lack of consistent, reliable inter-level coordination and questions around the sustainability of financial support for this donor-dependent system.

Following recognized best practices for improving specimen transport systems, ${ }^{9}$ the analysis recommended selecting and testing alternatives for district-to-central transport, including considering La Poste, a national parastatal postal company with wide operational presence down to district level. Following consultations with government partners, this recommendation was judged feasible; partnership with postal services has been shown to work well in similar settings. ${ }^{8}$

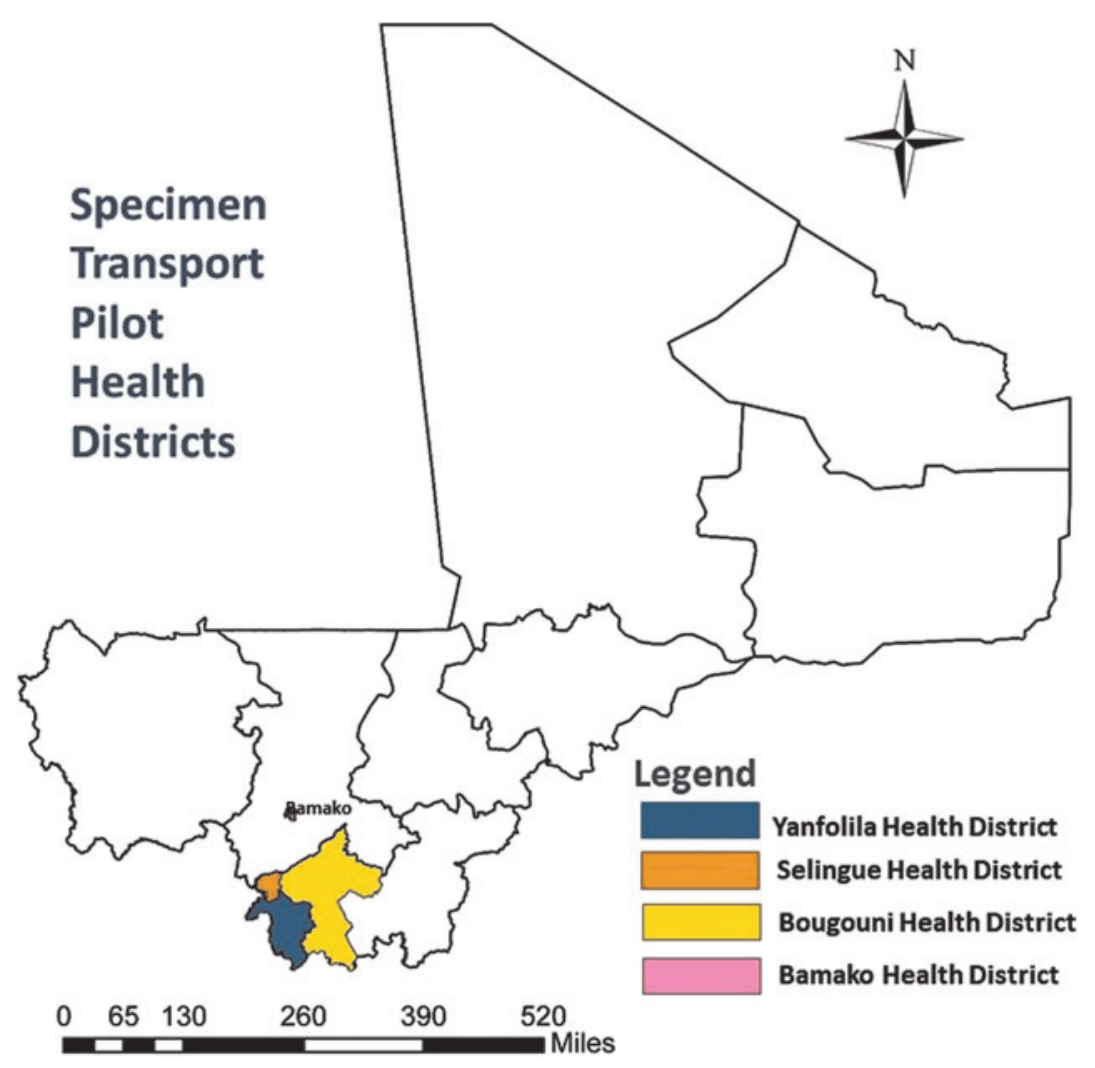

Figure 1. Study Sites. 
Catholic Relief Services/Mali, through its CDC-funded Djomi/GHSA laboratory strengthening project, developed a pilot to assess real-world feasibility of this approach. This article reports on the results of the intervention in terms of transit time, specimen quality, and cost. We also reflect on lessons learned and implications for programs aiming to strengthen specimen transport systems in similar resourceconstrained settings.

\section{Methods}

\section{Intervention}

The present 12-month pilot study compares the public transport and postal system for sending specimens from districts to the DNS, which dispatches them to reference laboratories. Following partner consultations and selection of the postal partnership for the pilot intervention, the study team approached La Poste to evaluate training needs and develop terms of service. A 1-year contract was established in study districts on the basis of a flat monthly fee of 750,000 West African CFA francs (US\$1,285) to send an unlimited number of specimens on demand. The project team provided training on sample handling to La Poste employees, as well as training and specimen transport material to health facilities. La Poste ensured transportation from the district capitals in its vehicles to the National Center for Immunization (CNI) in Bamako. The project team followed up weekly by telephone with La Poste and the National Public Health Research Institute (INRSP) to collect data.

\section{Study Sites}

Three health districts in the Sikasso region were selected for the study: Bougouni, Yanfolila, and Sélingué (Figure 1). These health districts cover 75 community health centers and were included because of their relatively high reported rates of epidemic-prone diseases.

For the community-to-district level, motorcycles are generally used to transport samples. For this study, it was decided that the post would deliver samples from districtto-central level (DNS) to allow for direct comparison of the public transport system to the postal system, as the public transport system operates only from district-to-central levels. Although it was acknowledged that community-todistrict transport suffers from challenges, these were beyond the scope of the intervention.

\section{Data Collection and Analysis}

Three indicators were of interest. One indicator was the average transport time for sending samples from the districts to Bamako (defined as the time from sample pick-up to the time that it arrives at the depot in Bamako). This information was communicated through real-time specimen transport system notification between La Poste and the study team and cross-checked with DNS's reception records. The second was quality of the sample upon reception (as defined by whether a sample is judged satisfactory and usable for testing upon arrival at laboratory-that is, not degraded, broken, missing, or otherwise compromised). The last indicator was cost.

The study compared these indicators from 2 databases-1 with data collected onsite during the study, and the second that aggregated retrospective data for the same interval of time from the previous period in the same districts (August 2015 to August 2016). Quantitative data (eg, number of samples sent, transfer time, etc) on performance of both systems were cross-checked across multiple ministry of health sources: case notification forms; epidemic-prone disease case registers in community health centers/districts; and DNS/laboratories specimen databases. Data were collected in Excel and analyzed in SPSS.24 using the Pearson $\mathrm{chi}^{2}(p=0.05)$.

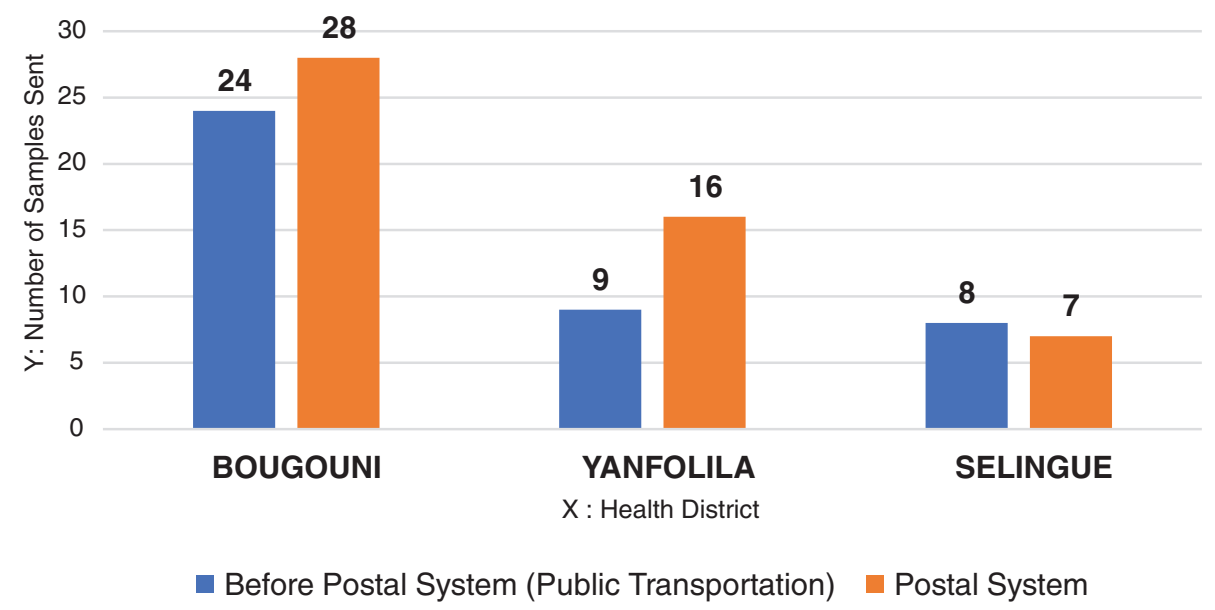

Figure 2. Number of Samples Sent from Health Districts Before the Study and with the Post. 
Table 1. Samples by Pathology and by District During and Before the Pilot Study

\begin{tabular}{|c|c|c|c|c|c|c|}
\hline \multirow[b]{2}{*}{ Period } & \multirow[b]{2}{*}{ Health Districts } & \multicolumn{4}{|c|}{ Type of Specimen } & \multirow[b]{2}{*}{ Total } \\
\hline & & Yellow Fever (Serum) & Meningitis (CSF) & Measles (Serum) & Polio (Fecal) & \\
\hline \multirow{4}{*}{$\begin{array}{l}\text { Public transport (prior to pilot) } \\
\text { (August } 2015 \text { to August 2016) }\end{array}$} & Bougouni & 2 & 10 & 6 & 6 & $24(59 \%)$ \\
\hline & Yanfolila & 0 & 3 & 1 & 5 & $9(22 \%)$ \\
\hline & Selingue & 1 & 4 & 1 & 2 & $8(20 \%)$ \\
\hline & Total & $3(7 \%)$ & $17(41 \%)$ & $8(20 \%)$ & $13(32 \%)$ & $41(100 \%)$ \\
\hline \multirow{4}{*}{$\begin{array}{l}\text { Postal system (August } 2016 \\
\quad \text { to August 2017) }\end{array}$} & Bougouni & 0 & 6 & 12 & 10 & $28(55 \%)$ \\
\hline & Yanfolila & 3 & 5 & 1 & 7 & $16(31 \%)$ \\
\hline & Selingue & 0 & 3 & 0 & 4 & $7(14 \%)$ \\
\hline & Total & $3(6 \%)$ & $14(27 \%)$ & $13(25 \%)$ & $21(41 \%)$ & $51(100 \%)$ \\
\hline
\end{tabular}

\section{Results}

Results were compared examining the data during the postal system pilot against the data from the preceding year in the same districts under the public transport system.

\section{Characteristics of Samples}

In total, 51 samples were sent during the study period compared to 41 the year before (Figure 2). There was a slight increase in the total number of samples sent during the pilot, but the difference in sample quantity sent is not significant, as we did not experience any outbreak either in the pre-pilot nor in the pilot periods that might cause a difference.

In both years, Bougouni health district had the largest number of samples (59\% before the pilot and 55\% during the pilot; Table 1). In terms of types of samples, cerebrospinal fluids for meningitis were the most-represented samples of suspected disease sent prior to the pilot at $41 \%$, while fecal samples for polio were the largest proportion of specimens sent during the pilot, also $41 \%$.

\section{Outcome Indicators}

Quality of samples on arrival at the laboratory was also measured. There was an improvement in the quality of samples received in reference laboratories, from $93 \%$ of samples arriving in good condition on public transport to $98 \%$ via the post. The difference was not statistically significant $(p=0.21)$.
During the pilot, most of samples were shipped to the DNS within 72 hours, while with the public transport system, the delivery time of more than half of samples exceeded the required transit time ( 72 hours). The mean transit time of samples by transport system was: Bougouni, 24 hours with la Post vs a week with public transport; Yanfolila, 24 hours with La post vs 2 weeks with public transport; Selingue, 1 day with La post vs 2 weeks with public transport. There was a statistically significant difference in the proportion of overall samples that arrived within 72 hours using the post (71\%) compared to public transport $(46 \%)$ and in those arriving after 72 hours $(p<0.018)$.

To calculate average overall cost of specimen shipment by public transport companies, the following costs were taken into account: the cost of the district health center's vehicles bringing samples to the bus station, and the cost of the National Health Directorate agent to pick up samples at the station in Bamako. The cost for La Poste includes all intermediary fees, as it directly picked up and dropped off samples from the districts to DNS. In total, the average overall per-specimen transport cost from district level was 21,337CFA for samples sent on public transport and 176,471 CFA via La Poste (Table 2).

\section{Discussion}

This study set out to evaluate the feasibility of a new partnership for integrated specimen transport from the district-to-national level using La Poste du Mali. There was a statistically significant difference on delivery of samples

Table 2. Cost Comparison for Contracting with Public Transport vs Postal System

\begin{tabular}{lcccc}
\hline & & Total Number of Average Cost in CFA & A \\
Period/Means of Transport & Type of Payment & Samples Received & per Sample Sent & Total Cost in CFA \\
\hline $\begin{array}{l}\text { Public transport companies } \\
\text { (August 2015 to August 2016) }\end{array}$ & Per unit sent & 41 & 21,337 & $(41 \times 21,337)=874,817$ \\
\hline $\begin{array}{l}\text { Postal system pilot (August 2016 } \\
\text { to August 2017) (One-year } \\
\text { contract signed with Poste du Mali) }\end{array}$ & $\begin{array}{c}\text { Monthly flat fee to send } \\
\text { unlimited samples } \\
(750,000 \text { CFA) }\end{array}$ & 51 & $176,471^{\mathrm{a}}$ & $9,000,000$ \\
\hline
\end{tabular}

\footnotetext{
${ }^{a}$ Average cost in CFA per sample sent $(176,471 \mathrm{CFA})=$ annual flat fee $(750,000 \times 12) / 51$.
} 
received in less than 72 hours, indicating positive correlation between use of the post and shorter duration of specimens in transit.

While it is clear that La Poste provided superior performance in terms of transit time, the difference in quality of samples on arrival was not found to be statistically significant. La Poste performed well and could be considered a viable alternative to existing specimen transport systems. Working and contracting with a single entity, La Poste, was also relatively simple, as compared to contracting with multiple public transport companies with varying levels of professionalism for different geographic and disease areas. Specimens could be sent individually and on demand, without requiring waiting for a "batch" of samples. With training and monitoring, La Poste was able to deliver specimens reliably. Operational feasibility of the partnership was apparent and is scalable based on La Poste's existing organization and widespread presence.

Evaluating the cost variable is complex. The study estimated that the overall cost of sending an epidemic-prone disease sample was 21,337 CFA on public transport versus 176,471 CFA per sample for the post. Public transport companies are clearly the more economical option (samples are sent per batch once a week). However, the fixed-fee contract mechanism used for the post is difficult to compare to the per-specimen scheme with public transport. The fixedfee approach used in this pilot (samples sent 3 time per week) may not be the appropriate solution for Mali, where specimen volumes are low. If a per-unit scheme could be negotiated with La Poste, or if the fixed-fee were calculated on the basis of historical average sample volumes, this cost could be optimized. Additionally, during epidemic periods, when there is a much greater flow of samples, a fixed-fee approach may present cost savings, and La Poste would have an advantage in terms of speed, quality, and cost. Economic modeling could assist in evaluating the impact of different epidemic scenarios on cost-efficiency of specimen transport systems.

The cost question has sustainability implications: A pilot with higher costs than existing systems is unlikely to find political support to bring to scale. Study stakeholders agreed that cost optimization was needed to account for low sample volumes. The Laboratory Sub-Commission is thus planning an exercise to quantify costs of the system and model different options of transporting specimens in epidemic and nonepidemic periods. The question of further scale-up requires national dialogue around effective costing and necessitates building consensus around a more integrated approach.

\section{Lessons Learned and Implications for Global Health Security}

- It is critical to optimize costs in developing the specimen transport system based on a thorough understanding of current costs. Mali had done a previous landscape analysis, but there had not been an economic evaluation or modeling done to help guide analysis of alternatives. This is now being conducted after the present pilot, but it would have been ideal to complete this analysis initially with all stakeholders.

- Just as important as ensuring an efficient means of transport are norms and resources to support correct specimen collection and packaging. While the project ensured support to the government to develop national guidelines, train personnel, and equip facilities with material complementary to this pilot, it is critical to engage the government around systems for reprovisioning all actors and developing more sustainable solutions-ideally, a national budget line for specimen transport material. Plans for pre-positioning of material at regional or district levels should also be defined; in Mali, a portion of stock was kept at each level to facilitate the rapid delivery.

- Close involvement of the national laboratory coordinating entity - in Mali's case, the Laboratory SubCommission - is necessary from the design phase. This is critical for selecting and refining a mechanism that has a real possibility of scale-up. To facilitate shared understanding of the current system and monitor changes, the current sample transport circuit should be mapped together with stakeholders. In Mali, the sample circuit was visually represented during a workshop, but GIS technology could assist decision making in visually representing pick-up and drop-off points and routes.

- Effective specimen transport systems are those that support an integrated, universal system of laboratory diagnostics rather than a fragmented approach.' However, in Mali as elsewhere, piecemeal funding continues to be provided by donors for different types of samples. Effort is needed to build consensus around integrating the system to handle all types of specimens. This requires collaboration among government, partners, and donors.

- Even if countries opt to retain a partial public-transport system, it could be optimized to improve performance. Developing and implementing standardized training of handlers and introducing or ensuring strong enforcement of quality provisions of existing contracts with public transport companies could reduce some of the most serious delays and biosecurity risks and would be preferable to making no change at all.

\section{Limitations}

The limited sample size does not necessarily allow extrapolation of feasibility to conditions in all areas, especially Mali's conflict-ridden north. However, La Poste's wide presence and standard functioning would allow it to operate in a similar way across much of Mali. Further evaluation is required to understand the specific constraints in 
the north and determine whether La Poste is a feasible service provider in that area. Another limitation is the different time periods used in this analysis, which could lead to bias in our results.

\section{Conclusion}

The study results affirmed that La Poste is an alternative means of transport and a potentially important partner that the Malian Ministry of Health should consider for rapid, secure specimen transport. Expanding the partnership with La Poste could alleviate delays and contribute to rapid availability of laboratory results. Laboratory system actors must build consensus on optimizing costs with low sample volumes while allowing for preparedness of the specimen transport systems for epidemics. This study adds to evidence for partnerships with nontraditional actors as a promising approach for addressing specimen transport system constraints.

\section{ACKNOWLedgMents}

This study was funded through CDC Grant No: NU2GGH001832, entitled Global Health Security Partner Engagement: Expanding Efforts and Strategies to Protect and Improve Public Health Globally.

\section{REFERENCES}

1. Summers A, Nyenswah TG, Montgomery JM, et. al. Challenges in responding to the Ebola epidemic-four rural counties, Liberia, August-November 2014. MMWR Morb Mortal Wkly Rep 2014;63(50):1202-1204.
2. Kilmarx PH, Clarke KR, Dietz PM, et al. Ebola virus disease in health care workers-Sierra Leone, 2014. MMWR Morb Mortal Wkly Rep 2014;63(49):1168-1171.

3. Joloba M, Mwangi C, Alexander $\mathrm{H}$, et al. Strengthening the tuberculosis specimen referral network in Uganda: the role of public-private partnerships. J Infect Dis 2016;213(Suppl 2): S41-S46.

4. Engel N, Davids M, Blankvoort N, Pai NP, Dheda K, Pai M. Compounding diagnostic delays: a qualitative study of pointof-care testing in South Africa. Trop Med Int Health 2015; 20(4):493-500.

5. World Health Organization. Joint External Evaluation Tool. 2d ed. Geneva: WHO; 2018. https://www.who.int/ihr/ publications/WHO_HSE_GCR_2018_2/en/. Accessed March 23, 2019.

6. Best M, Sakande J. Practical recommendations for strengthening national and regional laboratory networks in Africa in the global health security era. Afr J of Lab Med 2016;5(3):471.

7. Kebede Y, Fonjungo PN, Tibesso G, et al. Improved specimen-referral system and increased access to quality laboratory services in Ethiopia: the role of the public-private partnership. J Infect Dis 2016;213(Suppl 2):S59-S64.

8. African Society for Laboratory Medicine. Building Laboratory Capacity to Support the Global Health Security Agenda Assessment of Sample Referral Systems and Capabilities. Mali: ASLM; 2015.

9. Global Laboratory Initiative (GLI) Stop TB Partnership. GLI Guide to TB Specimen Referral Systems and Integrated Networks. Geneva: Stop TB Partnership; 2018. http://www.stoptb.org/ wg/gli/assets/documents/gli_guide_specimens_web_ready.pdf. Accessed March 23, 2019.

Address correspondence to: Hamadoun Kassambara Chief of Party Project Djomi/Global Health Security Agenda Catholic Relief Services Bamako BP E3256 Mali

Email: hamadoun.kassambara@crs.org 